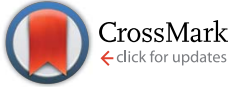

Cite this: RSC Adv., 2017, 7, 8542

Received 3rd December 2016 Accepted 20th December 2016

DOI: 10.1039/c6ra27709h

www.rsc.org/advances

\title{
The construction of electrochemical chiral interfaces using hydroxypropyl chitosan
}

\author{
Zhenliang Li, Zunli Mo, ${ }^{*}$ Shujuan Meng, Huhu Gao, Xiaohui Niu, Ruibin Guo \\ and Taibao Wei
}

A simple chiral electrochemical sensor based on hydroxypropyl chitosan (HPCS) covalently bound to multiwalled carbon nanotubes (MWCNTs) was developed for the recognition of mandelic acid (MA) enantiomers. HPCS preferably combines with (S)-MA over (R)-MA, which was attributed to the favorable host-guest interactions between HPCS and the MA enantiomers. In addition, the recognition efficiency was significantly increased after hydroxypropyl groups were grafted onto chitosan (CS), which may be due to a stereo-hindrance effect. Moreover, the electrochemical recognition of amino acid enantiomers was investigated; the functional groups of the enantiomers play an important role in the enantioselective discrimination.

\section{Introduction}

The chiral recognition of active synthetic and natural compounds is a fundamental field, which is attracting increasing attention, particularly in the enantioselective recognition of chiral drugs. ${ }^{1} \mathrm{~A}$ critical step in the fabrication of chiral electrochemical sensors is to build a chiral surface with recognition sites for discriminating enantiomers; the modified chiral selector plays a key role in the fabrication of the chiral surface. ${ }^{2}$ Thus far, various chiral selectors have been applied in enantioselective recognition such as bovine serum albumin, ${ }^{3,4}$ human serum albumin,,$^{5,6}$ deoxyribonucleic acid (DNA), sodium alginate, ${ }^{8} \beta$-cyclodextrin ${ }^{9}$ and amino acids. ${ }^{10}$

Chitosan (CS) is similar to DNA with a double helix structure; its wide substrate scope and significant selectivity are attributed to the existence of a large amount of chiral sites. ${ }^{11}$ CS was prepared by alkaline deacetylation of chitin with a high degree of deacetylation, it has poor solubility in water but is soluble in aqueous dilute acids. ${ }^{12}$ The limited solubility of CS restricts its use in chiral electrochemical sensors; ${ }^{13}$ however, there is an enormous potential for structural modification to impart special chemical functions and properties. Apart from the hydroxyl groups, there are several ways to chemically modify the amino groups in CS. ${ }^{14} \mathrm{X}$. Q. Chen et al. (2015) constructed a chiral electrochemical sensor based on CS functionalized multi-walled carbon nanotubes (MWCNTs) used for the discrimination of tryptophan (Trp) enantiomers. ${ }^{15}$ Y. Kong et al. (2015) developed a novel enantioselective system to enantioselectively recognize

Key Laboratory of Eco-Environment-Related Polymer Materials, Ministry of Education of China, Key Laboratory of Polymer Materials of Gansu Province, College of Chemistry and Chemical Engineering, Northwest Normal University, Lanzhou 730070, P. R. China.E-mail: mozlnwnu2010@163.com
Trp based on sulfonated chitosan and the recognition efficiency was improved remarkably because of the introduction of the alkyl sulfonate, which generated significant steric hindrance in the formation of intermolecular hydrogen bonds in the enantioselective recognition system. ${ }^{16}$ Hydroxypropyl chitosan (HPCS) is regarded as an important functional derivative of chitosan. The water solubility is enhanced when the hydroxypropyl group is introduced into CS. ${ }^{17}$ Moreover, there is a possible role in the discrimination of chiral enantiomers due to the introduced hydroxypropyl groups leading to the possibility of different affinities between CS and enantiomers. ${ }^{18}$

MA enantiomers are important structural units of several natural products and drug molecules, particularly, $(R)$-MA serves as chiral synthon for the synthesis of drugs, such as semisynthetic penicillin and cephalosporin. ${ }^{19} \mathrm{Y}$. Z. Fu et al. developed a strategy for the recognition of mandelic acid (MA) enantiomers in the presence of $\mathrm{Zn}$ (II) ions based on chiral ligand exchange. ${ }^{20} \mathrm{E}$. Zor et al. reported an electrochemical biosensor for the discrimination of MA enantiomers based on a three-points interaction model, which has a shorter response time and higher recognition efficiency than the chiral ligand exchange model. ${ }^{21}$ However, a fast electrochemical chiral sensor used for the discrimination of MA enantiomers based on MWCNTs-HPCS has not been reported to date.

In this study, the water-soluble chitosan derivative, HPCS was prepared through amino protection using benzaldehyde in order to selectively introduce the hydroxypropyl group. HPCS was linked to the carboxylic multi-walled carbon nanotubes (MWCNTs-COOH) through covalent bonding after deprotection of the amino groups. The MWCNTs-HPCS was used to construct an electrochemical sensor used for the stereoselective recognition of MA enantiomers via cyclic voltammetry (CV) and the results were satisfactory. 


\section{Experimental}

\section{Reagents and materials}

Chitosan (with a degree of deacetylation of 95\%). $N$-Hydroxy succinimide (NHS, 98\%) and $N$-(3-dimethylamino-propyl)- $N^{\prime}$-ethylcarbodiimide hydrochloride (EDC, 99\%) were purchased from Aladdin Industrial Corporation. 1,2-Epoxypropane was supplied by Sinopharm Chemical Reagent Co., Ltd. Tetramethylammonium hydroxide and benzaldehyde were supplied by Tianjing Kaixing Chemical Reagent Co., Ltd (Tianjing, China). The multi-walled carbon nanotubes (MWCNTs) were obtained from Shenzhen Bier technology Co., Ltd (Shenzhen, China). The MA enantiomers were purchased from Energy Chemical Corporation. All chemicals were of analytical grade and used without further purification. Ultrapure water was used throughout the experiments.

\section{Apparatus}

All electrochemical measurements were carried out on a CHI60E (Shanghai Chenhua Instruments Co., China). A threeelectrode system included a glassy carbon electrode (GCE, $\Phi=3$ $\mathrm{mm}$ ) as the working electrode, a platinum wire as the auxiliary electrode, and a saturated calomel electrode as the reference electrode. The morphology and functional groups of the MWCNTs-COOH, HPCS and MWCNTs-HPCS hybrids were recorded using a JSM-6701F cold field emission scanning electron microscope (Japan). Fourier-transform infrared (FT-IR) spectra were obtained using a QUINOX55 spectrometer. X-ray photoelectron spectroscopy (XPS) analysis was characterized using an Axis Ultra spectrometer.

\section{Preparation of hydroxypropyl chitosan}

$0.5 \mathrm{~g}$ of CS was added into $100 \mathrm{~mL}$ of an acetic acid solution (1 $\mathrm{wt} \%$ ) in a round-bottom flask. A mixture of $2.7 \mathrm{~mL}$ of benzaldehyde and $15 \mathrm{~mL}$ of ethanol was added and the CS solution was stirred at room temperature for $3 \mathrm{~h}$. The mixture was adjusted to $\mathrm{pH}=7 \mathrm{using}$ sodium hydroxide solution, filtered and washed with acetone and absolute ethanol to remove the unreacted benzaldehyde, and the $N$-benzylidene chitosan (B-CS) was obtained after being dried at $40{ }^{\circ} \mathrm{C}$.

B-CS $(0.3 \mathrm{~g})$ was basified using $2.0 \mathrm{~g}$ of a sodium hydroxide aqueous solution ( $35 \mathrm{wt} \%$ ) in an isopropanol solution $(4 \mathrm{~mL})$ for $12 \mathrm{~h}$. Alkali CS was incorporated into $4 \mathrm{~mL}$ of isopropanol, 5 drops of tetramethylammonium hydroxide $(25 \mathrm{wt} \%)$ and $3 \mathrm{~mL}$ of propylene oxide were added, and the mixture was heated at $45{ }^{\circ} \mathrm{C}$ for $12 \mathrm{~h}$. The reaction mixture was adjusted to $\mathrm{pH}=7$ using hydrochloric acid (20 wt\%), cooled and washed several times with acetone. Afterwards the products were soaked in 0.25 $\mathrm{N}$ hydrochloric acid/ethanol solution overnight as the deprotection step and white HPCS powder was obtained after filtration and vacuum drying at $50{ }^{\circ} \mathrm{C}$ for $12 \mathrm{~h} .{ }^{22}$

\section{Preparation of hydroxypropyl chitosan functionalized multi- walled carbon nanotubes}

The MWCNTs-COOH was synthesized as described previously. ${ }^{23}$ First, $0.03 \mathrm{~g}$ of HPCS was dissolved in $5 \mathrm{~mL}$ of an acetic acid solution ( $1 \mathrm{wt} \%$ ). Next, $0.006 \mathrm{~g}$ of MWCNTs-COOH was dispersed in $2 \mathrm{~mL}$ of ultrapure water via ultrasonication. $0.08 \mathrm{~g}$ of EDC and $0.2 \mathrm{~g}$ of NHS were added to the abovementioned solution. Subsequently, the HPCS solution was added under continuous stirring for $24 \mathrm{~h}$. The product was isolated by filtration and dried under vacuum at $60{ }^{\circ} \mathrm{C} .{ }^{24}$ The synthesis process used to prepare hydroxypropyl chitosan is shown in Fig. 1.

\section{Fabrication of the MWCNTs-HPCS chiral sensor}

Prior to the experiment, the glassy carbon electrode (GCE, $\Phi=3$ $\mathrm{mm}$ ) was polished with $1.0,0.3$ and $0.05 \mu \mathrm{m}$ alumina slurry. Afterwards, the GCE was sonicated in ethanol and ultrapure water and dried in air. Then, $6 \mathrm{~mL}$ of a homogeneous MWCNTsHPCS dispersed solution was dropped on the surface of the freshly polished GCE and then dried using an infrared lamp. As a comparison, a MWCNTs-CS/GCE was prepared using the same procedure. The fabrication process is illustrated in Scheme 1.

\section{Results and discussion}

\section{Characterization of the prepared MWCNTs-HPCS}

The surface morphologies of the MWCNTs-HPCS films at different stages were studied using scanning electron microscopy (SEM). As shown in Fig. 2A, the MWCNTs-COOH have a kinkled tubular structure and are intertwined with each other. Compared with MWCNTs-COOH, the diameter of MWCNTsHPCS was changed, which indicated that the HPCS was grafted onto the sides of the MWCNTs (Fig. 2B).

As shown in Fig. 3, the products of HPCS and MWCNTsHPCS were confirmed via the FT-IR spectra of CS, HPCS, MWCNTs-COOH and MWCNTs-HPCS. For CS (curve a), the stretching vibrations of $\mathrm{O}-\mathrm{H}$ are detected at approximately 3452 $\mathrm{cm}^{-1}$, and the two characteristic peaks located at $1629 \mathrm{~cm}^{-1}$ and $1617 \mathrm{~cm}^{-1}$ are also observed, which can be assigned to the stretching vibrations of $\mathrm{C}=\mathrm{O}$ and the bending vibrations of $\mathrm{N}-$ $\mathrm{H}$, respectively. ${ }^{25}$ HPCS exhibits similar absorption peaks to those of CS (curve b), the broad peak at around $3410 \mathrm{~cm}^{-1}$ was ascribed to the stretching vibrations of $\mathrm{O}-\mathrm{H}$ due to intermolecular or intramolecular hydrogen bonding. The stretching vibration enhancement of $-\mathrm{CH}_{2}$ - was located at $2887 \mathrm{~cm}^{-1}$. Moreover, the characteristic peaks of $-\mathrm{CH}_{3}$ appeared at 2970 $\mathrm{cm}^{-1}$ and $1382 \mathrm{~cm}^{-1}$. In addition, the stronger peak of $\mathrm{C}-\mathrm{O}$ moved to $1072 \mathrm{~cm}^{-1}$. This evidence suggests that the hydroxylpropyl group had been linked to $\mathrm{CS} .{ }^{26}$ For MWCNTs-COOH (curve c), the characteristic peaks of MWCNTs-COOH were centered at $1713 \mathrm{~m}^{-1}(\mathrm{C}=\mathrm{O}), 1370 \mathrm{~cm}^{-1}(\mathrm{C}-\mathrm{O})$ and $3439 \mathrm{~cm}^{-1}$ $(-\mathrm{OH})$. The peak of $\mathrm{C}=\mathrm{O}$ stretching for MWCNTs-COOH $(1713$ $\mathrm{cm}^{-1}$ ) shifts to $1698 \mathrm{~cm}^{-1}$ due to the amidization reaction (curve d). In addition, a new strong band at $1629 \mathrm{~cm}^{-1}$ appeared in curve $d$ of the MWCNTs-HPCS due to the formation of -NHCO-, which indicated that the hydroxypropyl group was grafted onto the MWCNTs-COOH. ${ }^{27,28}$

HPCS was grafted onto MWCNTs-COOH covalently, which can be confirmed from the FT-IR spectra. The full XPS spectrum 

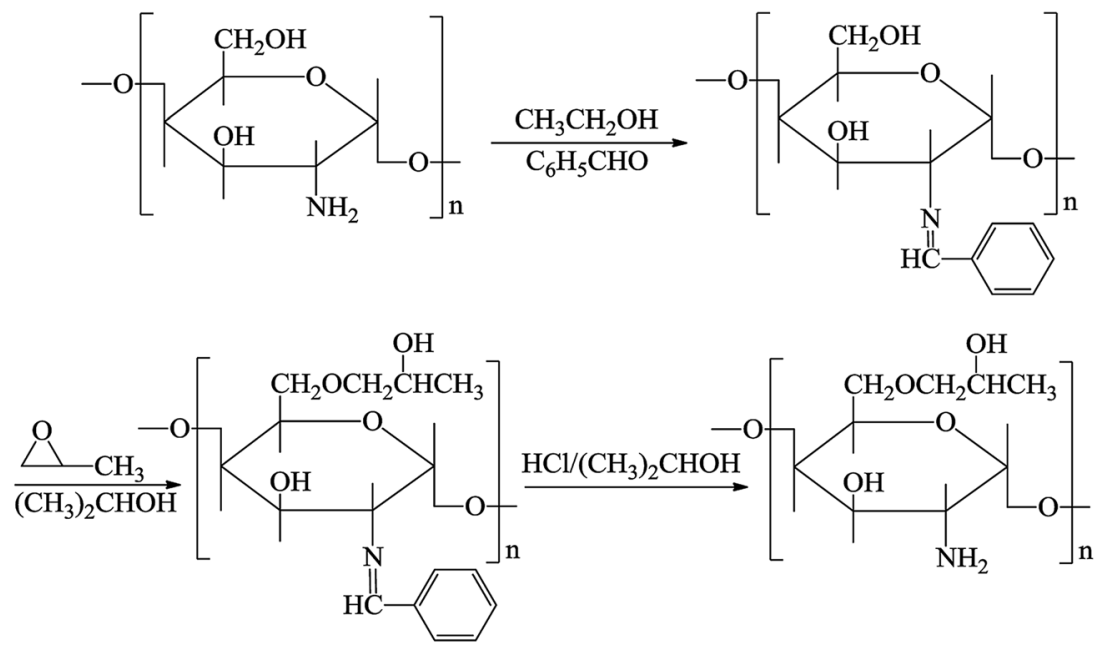

Fig. 1 The procedures used for the preparation of hydroxypropyl chitosan.

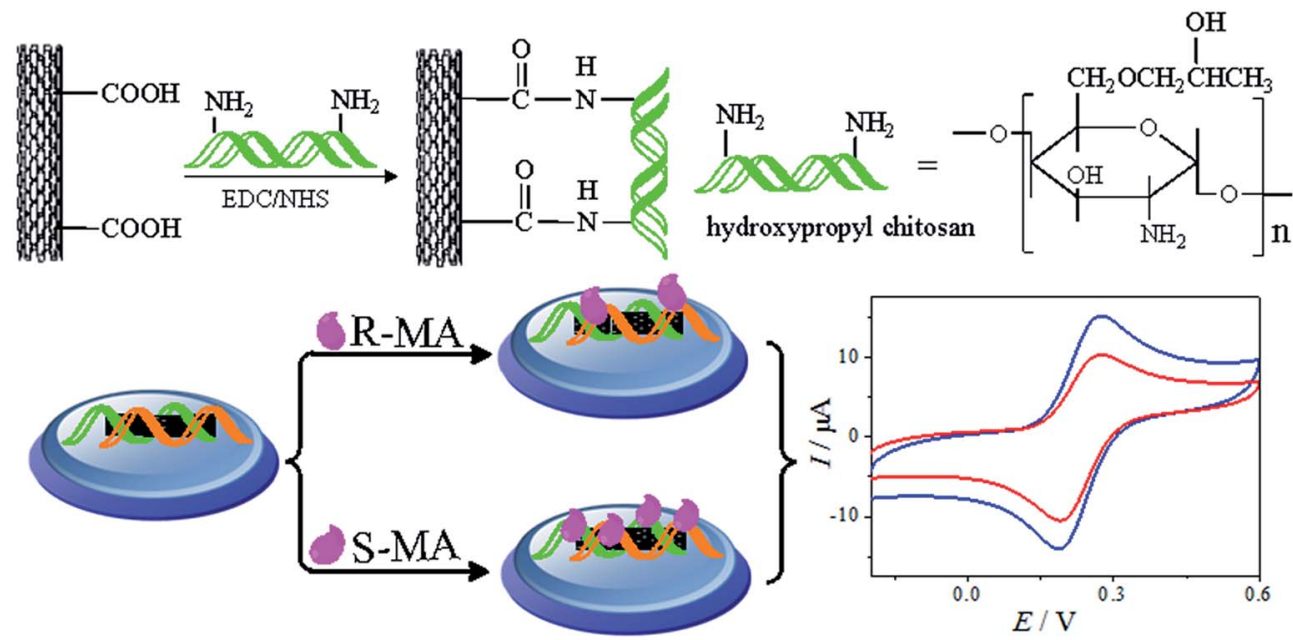

Scheme 1 A schematic of the MWCNTs-HPCS chiral sensor fabrication and the electrochemical responses to MA enantiomers.

of MWCNTs-HPCS is shown in Fig. 4A. The XPS survey scan of the MWCNTs-HPCS indicates the presence of C $1 \mathrm{~s}(286.3 \mathrm{eV}), \mathrm{O}$ 1s $(533 \mathrm{eV})$ and $\mathrm{N} 1 \mathrm{~s}(401 \mathrm{eV})$ peaks. The high resolution XPS C 1s is shown in Fig. 4B; the peak located at $286.3 \mathrm{eV}$ was due to the $\mathrm{C}-\mathrm{N}$ bond of HPCS. Moreover, the peaks at $287.8 \mathrm{eV}(\mathrm{O}=\mathrm{C}-$ $\mathrm{NH})$ and $289.2 \mathrm{eV}(\mathrm{O}=\mathrm{C}-\mathrm{O})$ were observed. More importantly, the peak observed at $287.8 \mathrm{eV}$, which originated from the amidation reaction between MWCNTs-COOH and HPCS, suggests that HPCS was successfully grafted onto the MWCNTs$\mathrm{COOH}^{29,30}$

\section{Electrochemical recognition of MA enantiomers with MWCNTS-HPCS}

$\mathrm{CV}$ was performed to investigate the electrochemical properties of the MWCNTs-HPCS modified GCE in $5.0 \mathrm{mM}\left[\mathrm{Fe}(\mathrm{CN})_{6}\right]^{4-/ 3-}$ containing $0.1 \mathrm{M} \mathrm{KCl}$ as the electroactive probe couple ${ }^{31,32}$ (Fig. 5A). The $\mathrm{CV}$ of the bare GCE in $\left[\mathrm{Fe}(\mathrm{CN})_{6}\right]^{4-/ 3-}$ was also carried out for comparison (a), a couple of well-defined redox peaks appeared at the MWCNTs-COOH/GCE, which were caused by the redox transition between the electroactive probe couple. The peak currents at the MWCNTs-COOH/GCE increased significantly, and the $\Delta I_{\mathrm{P}}$ was increased due to the high conductivity of the introduced MWCNTs (b). Moreover, the peak currents of MWCNTs-HPCS/GCE (d) were lower than those of MWCNTs-CS (c) due to the introduction of the hydroxylpropyl group. The result suggests that the HPCS provides more hindrance to the electronic transition of the electrode. ${ }^{33}$

Electrochemical impedance spectroscopy (EIS) was applied to investigate the interfacial properties of the modified electrodes. Moreover, the equivalent circuit was provided in Fig. 5B. The bare GCE has the biggest semicircle, and the $R_{\text {et }}$ value of the bare GCE was about $1073 \Omega$. However, the $R_{\text {et }}$ value of the MWCNTs-COOH was $114 \Omega$, which acts as a conducting bridge, and it was found that the value of the charge transfer resistance was significantly lower than the other electrode. Moreover, as for the HPCS $(167 \Omega)$, the value of resistance was larger than CS $(143 \Omega)$ due to the introduction of the hydroxypropyl groups, 


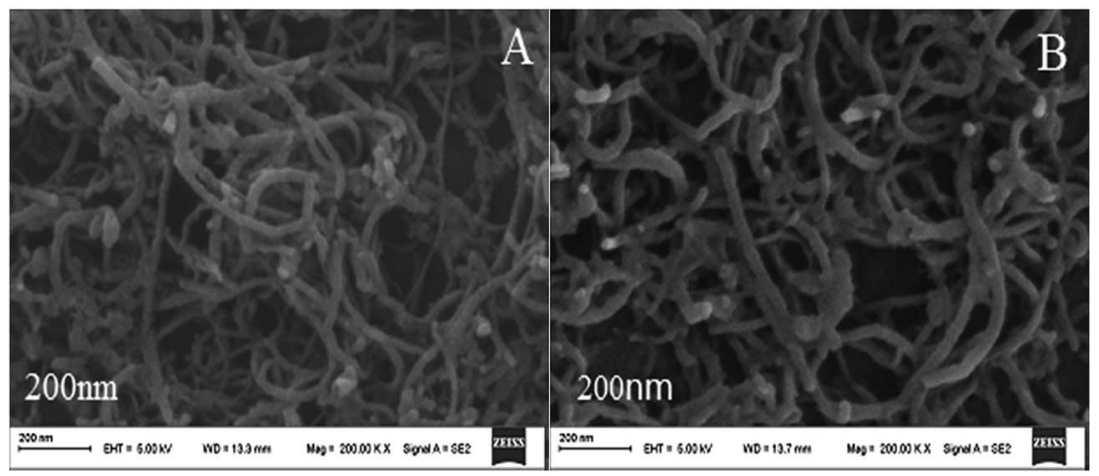

Fig. 2 SEM images of (A) MWCNTs-COOH and (B) MWCNTs-HPCS.

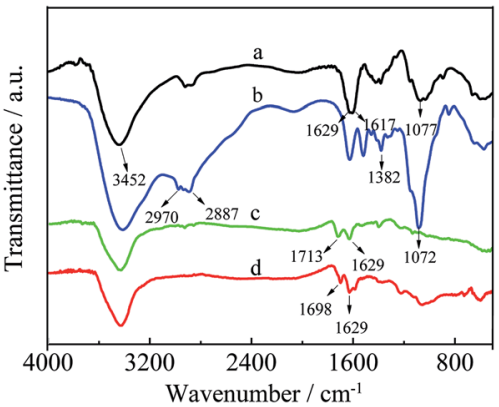

Fig. 3 The FT-IR spectra of CS (a), HPCS (b), MWCNTs-COOH (c) and MWCNTs-HPCS (d).

which was attributed to the obstruction of electron transfer from HPCS. The results from EIS and CV were confirmed with each other.

$\mathrm{CV}$ was used to study the electrochemical properties of the modified electrodes, and $\left[\mathrm{Fe}(\mathrm{CN})_{6}\right]^{4-/ 3-}$ was applied as the electroactive probe to record the peak currents of the different modified electrodes. The current difference $\left(\Delta I_{\mathrm{p}}\right)$ of the $\left[\mathrm{Fe}(\mathrm{CN})_{6}\right]^{4-/ 3-}$ redox couple indicates the changes in the progress of GCE modification. Fig. 6 shows the CV of $(R)$ - and $(S)$-MA at the different electrochemical interfaces. The MA enantiomers exhibited completely similar responses at the bare GCE (Fig. 6A), suggesting that the bare GCE was incapable of recognizing the MA enantiomers due to the absence of a chiral selector. Similarly, there was no significant difference in the peak currents $\left(I_{\mathrm{p}}\right.$ ) observed in the CV of the MWCNTs (Fig. 6B). When compared with CS as a chiral selector (Fig. 6C), discernable differences in the $I_{\mathrm{p}}$ appeared in the CV of the HPCS modified GCE (Fig. 6D); HPCS exhibited a higher affinity for $(S)$ MA than $(R)$-MA. It was noteworthy that a remarkable difference can be observed from the $I_{\mathrm{p}}$ based HPCS, which may be attributed to the stereo-hindrance of the hydroxypropyl groups. In addition, after the hydroxypropyl groups were grafted onto the CS, the hydrophility of HPCS was greatly improved, which lead to the formation of hydrogen bonds between the MA enantiomers and HPCS. Furthermore, the hydrogen bonds were more easily formed between (S)-MA and HPCS compared with $(R)$-MA, which was attributed to the different stereoisomeric configurations of the MA enantiomers. The results indicate that the peak current ratio $\left(I_{R-\mathrm{MA}} / I_{S-\mathrm{MA}}\right)$ with the HPCS chiral interfaces (1.48) was significantly higher than CS (1.13). The possible recognition mechanism is shown in Fig. 7.

\section{Analysis of single MA enantiomers}

The current response of the MWCNTs-HPCS modified GCE to $(R)$-MA and $(S)$-MA at various concentrations is shown in Fig. 8A. Along with the $(R)$-MA concentration increasing from 2 to $10 \mathrm{mM}$, more and more of the $(R)$-MA interact with the surface of the MWCNTs-HPCS/GCE, and the peak current gradually decreases. The linear relationship between peak current and the concentration of $(R)$-MA is shown in the inset of Fig. 8A. The
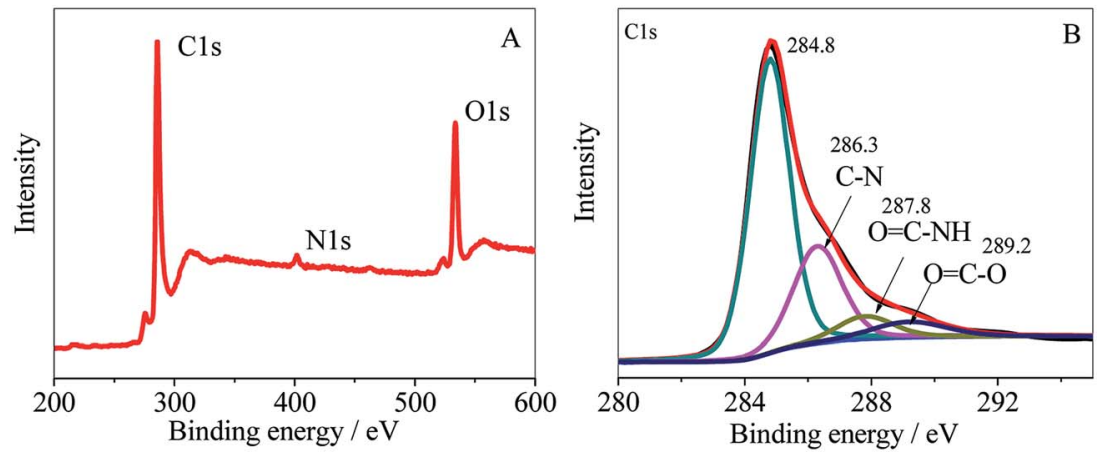

Fig. 4 The XPS spectrum of the (A) survey scan and (B) C 1s region of the MWCNTs-HPCS composite. 

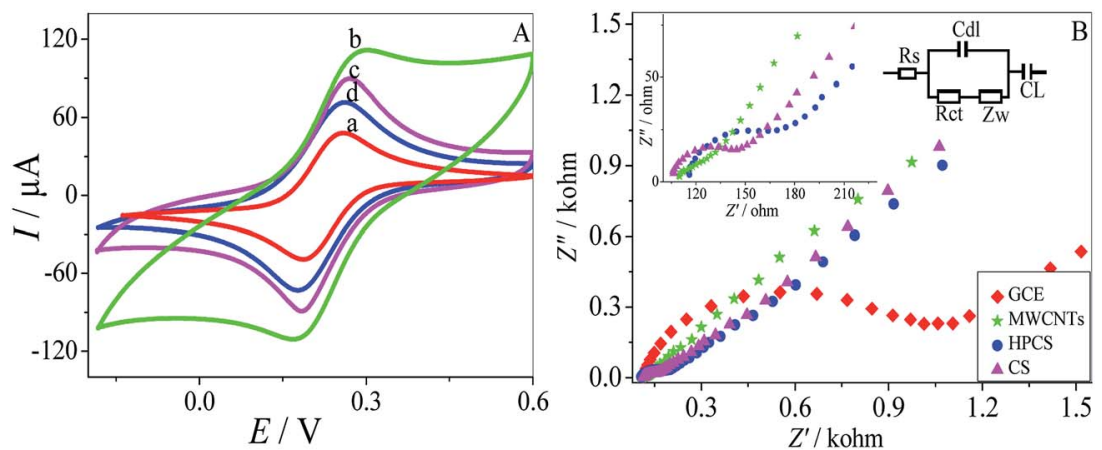

Fig. 5 (A) $\mathrm{CV}$ of $5.0 \mathrm{mM}\left[\mathrm{Fe}(\mathrm{CN})_{6}\right]^{4-13-}$ containing $0.1 \mathrm{M} \mathrm{KCl}$ at the bare GCE (a), MWCNTs-COOH/GCE (b), MWCNTs-CS/GCE (c) and MWCNTs-HPCS (d). (B) Nyquist plots at the bare GCE, MWCNTs-COOH, MWCNTs-HPCS and MWCNTs-CS. Inset: magnified Nyquist plots at the MWCNTs, HPCS and CS (left); equivalent circuit (right).
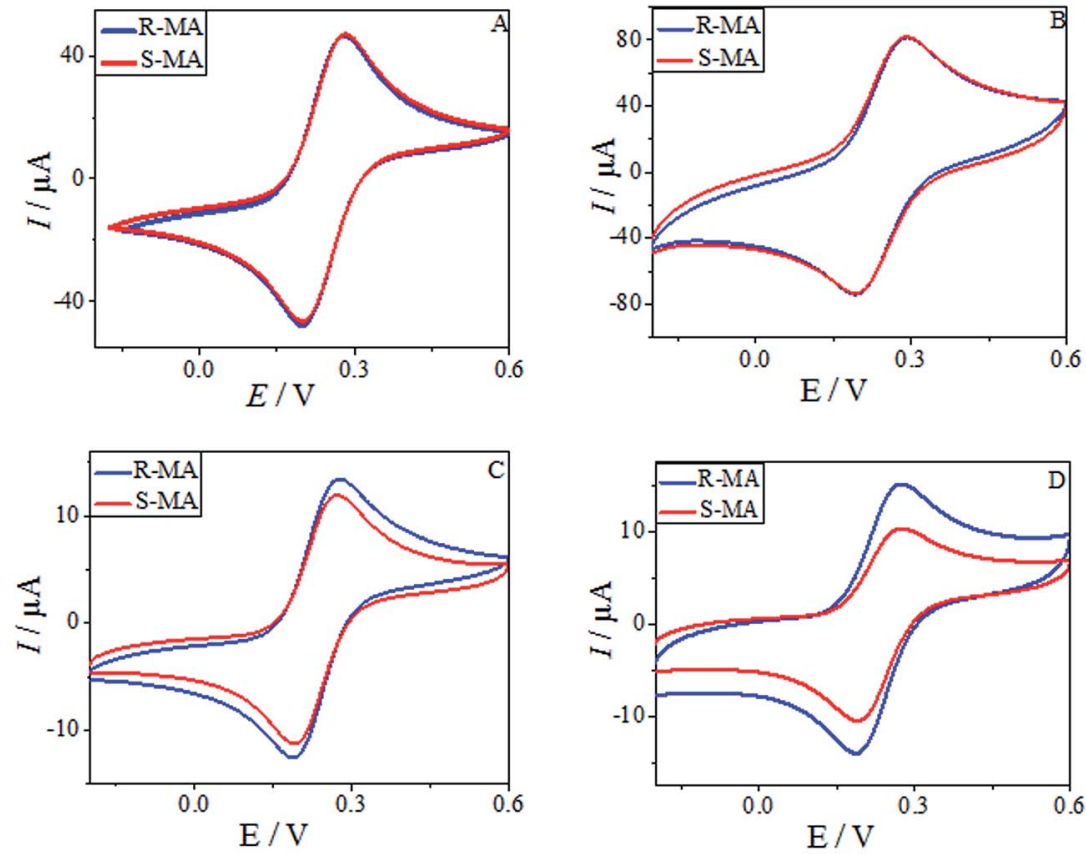

Fig. $6 \mathrm{CV}$ for the redox reactions of $10 \mathrm{mM}$ MA on the different electrodes in $0.2 \mathrm{M}$ PBS buffer solution (pH 7.0 ) containing $5.0 \mathrm{mM}$ $\left[\mathrm{Fe}(\mathrm{CN})_{6}\right]^{4-13-}$. (A) Bare GCE, (B) MWCNTs, (C) MWCNTs-CS and (D) MWCNTs-HPCS.

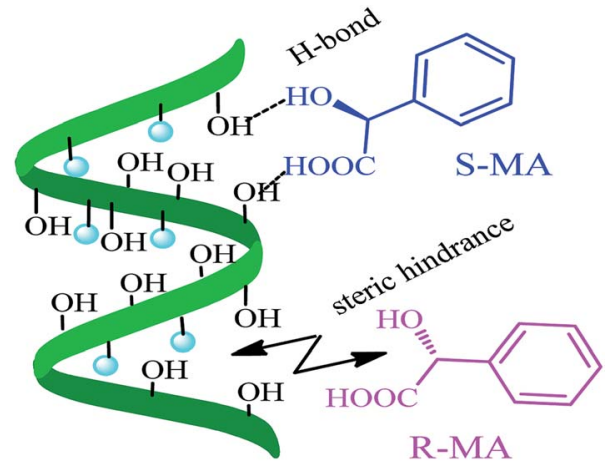

Fig. 7 The chiral recognition mechanism between MWCNTs-HPCS and the MA enantiomers. peak current decreased upon increasing the $(S)$-MA concentration from 2 to $10 \mathrm{mM}$ and the relationship of $(S)$-MA concentration and peak current is illustrated (Fig. 8B). The linear regression equations for $(R)$-MA and $(S)$-MA can be presented: $I_{\mathrm{p}}$ $(\mu \mathrm{A})=-1.14 c+20.06\left(R^{2}=0.9963\right)\left(\right.$ curve A) and $I_{\mathrm{p}}(\mu \mathrm{A})=$ $-0.66 c+17.18\left(R^{2}=0.9930\right)$ (curve $\left.\mathrm{B}\right)$, respectively. In addition, the $(R)$-MA and $(S)$-MA enantiomers can be discriminated by the MWCNTs-HPCS/GCE in this concentration range.

\section{Optimization of the experimental conditions}

The $\mathrm{pH}$ of the solution has an influence in enantiospecificity of the MA enantiomers. The effect of $\mathrm{pH}$ on the enantioselective recognition was studied from 6.0 to 8.0 in $10 \mathrm{mM} \mathrm{MA}$, as shown in (Fig. 9A) and the maximum $\Delta I$ value appeared at pH $7.0(\Delta I=$ $\left.I_{R \text {-MA }}-I_{S \text {-MA }}\right)$. Consequently, pH 7.0 was the optimized value for 

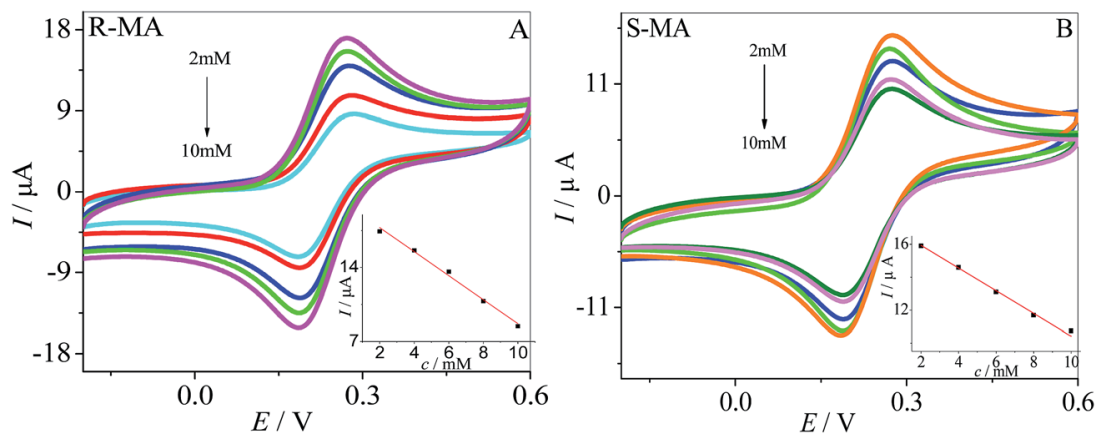

Fig. $8 \mathrm{CV}$ images of (R)-MA (A) and (S)-MA (B) at MWCNTs-HPCS modified GCE in 0.2 M PBS buffer solution (pH 7.0) containing 5.0 mM $\left[\mathrm{Fe}(\mathrm{CN})_{6}\right]^{4-13-}$. Inset: linear relationship of the concentration of MA enantiomers and $I_{\mathrm{p}}$ of $\mathrm{CV}$ corresponding.
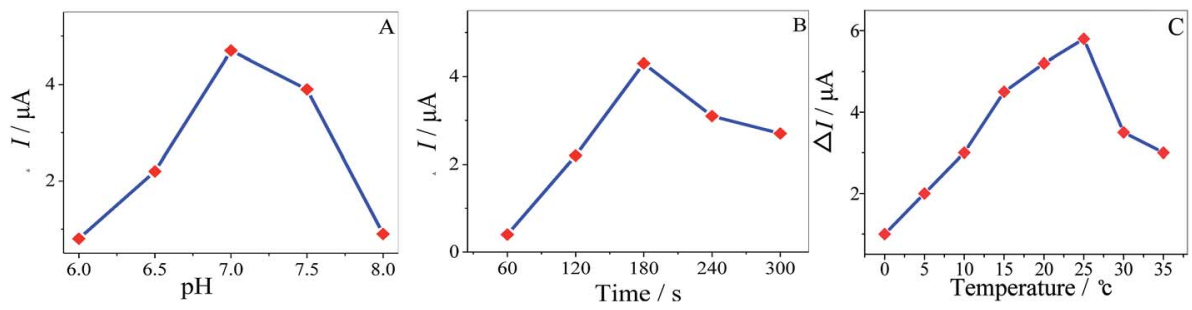

Fig. 9 The influence of $\mathrm{pH}(\mathrm{A})$, incubation time $(\mathrm{B})$ and temperature $(\mathrm{C})$ on the current response of the MWCNTs-HPCS in $5.0 \mathrm{mM}\left[\mathrm{Fe}(\mathrm{CN})_{6}\right]^{4-/ 3-}$ containing 10 mM MA enantiomers.

chiral recognition. It was important to investigate the incubation time of the MWCNTs-HPCS/GCE in the MA enantiomers; the peak currents increased with the incubation time, as shown in (Fig. 9B), and the maximum $\Delta I$ value appeared at $180 \mathrm{~s}$. The greater water solubility of HPCS may play an important role in enantioselective recognition. Similar chiral sensing platforms are reported in Table 1. The influence of temperature on the $\Delta I$ value at the MWCNTs-HPCS based chiral interfaces is shown in Fig. 9C. Clearly, the highest $\Delta I$ value was achieved at $25{ }^{\circ} \mathrm{C}$. When the temperature was below $10^{\circ} \mathrm{C}, \mathrm{H}$-bond formation was favorable, which has a close relationship with angles of the relative orientation. Then, the kinetic energy increased with temperature, and thus the increasing orientational disorder broke the $\mathrm{H}$-bonds, ${ }^{34-36}$ which resulted in a low recognition efficiency.

\section{Electrochemical discrimination of the amino acid enantiomers}

The HPCS, as a chiral selector, was further applied to the chiral discrimination of the enantiomers of phenylalanine (Phe) and
Trp under the same conditions, and the results are shown in Fig. 10. The Phe and Trp enantiomers show differences in $I_{\mathrm{p}}$ based on the HPCS chiral interfaces; the $I_{\mathrm{D}-\operatorname{Trp}} / I_{\mathrm{L}-\mathrm{Trp}}$ value for the Trp enantiomers (1.14) was much higher than those of Phe (1.05), which suggests that HPCS was capable of recognizing the Trp and Phe enantiomers as well as MA. According to the threepoint interaction model, the chemical structures of MA, Trp and Phe play an important role in the enantioselective discrimination process. As shown in Fig. 11, Trp enantiomers provide more steric hindrance due to the benzopyrole group during the formation of hydrogen bonds. Compared with the Phe enantiomers, the recognition efficiency for the Trp isomers decreased significantly. The chiral sites of MA are different to the Phe and Trp enantiomers; the hydroxy and carboxyl groups are linked to the chiral carbon of MA. In addition, the functional groups of MA more easily form hydrogen bonds. ${ }^{37}$ Furthermore, there were many kinds of electron-donating groups in the enantiomers, such as benzopyrole and benzene; the distance between the functional group and chiral carbon is an important factor during the enantioselective discrimination process. $^{38}$

Table 1 The electrochemical methods used for recognition of MA enantiomers reported to date ${ }^{a}$

\begin{tabular}{lllc}
\hline Modified electrode & Chiral recognition principle & $\mathrm{pH}$ & Interaction time \\
\hline L-Cys-Au & Ligand exchange model & 5.5 & $10 \mathrm{~min}$ \\
GLOB/rGO/GCE & Three-point interaction model & 7.2 & 1440 min \\
MWCNTs-HPCS/GCE & Three-point interaction model & 7.0 & 3 min
\end{tabular}

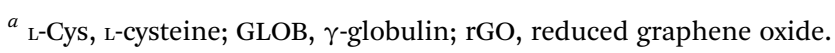



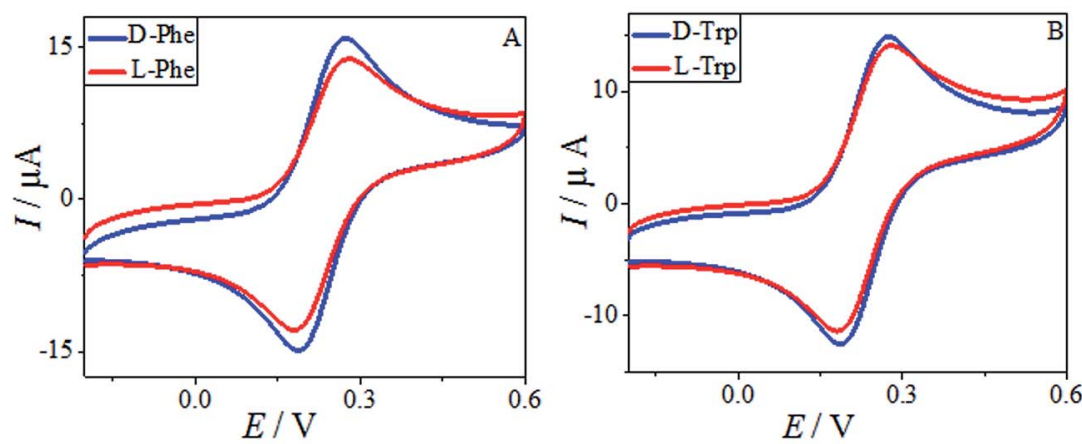

Fig. 10 The CV of $10 \mathrm{mM}$ Phe enantiomers (A) and Trp enantiomers (B) in $0.2 \mathrm{M} \mathrm{PBS}$ buffer solution (pH 7.0) containing 5.0 mM [Fe(CN) $]^{4-/ 3-}$.<smiles>NC(Cc1ccccc1)C(=O)O</smiles>

Fig. 11 The chemical structures of phenylalanine (Phe), mandelic acid (MA) and tryptophan (Trp).

\section{Chiral recognition of MA enantiomers in a racemic solution}

We tried to develop a simple method for the discrimination of one enantiomer in the racemic solution, in this manner, the MWCNTs-HPCS/GCE was used for determination of the mixture of $(S)$ - and $(R)$-MA. The peak currents of the MA enantiomers were recorded as a ratio of $(S)$ - and $(R)$-MA $(0: 100,20: 80,40: 60,60: 40,80: 20$ and $100: 0, \mathrm{v} / \mathrm{v})$ via $\mathrm{CV}$ (Fig. 12). It is important for the chiral discrimination in racemic mixtures of chiral compounds, especially chiral drugs, and it is highly desirable for the determination of the chiral enantiomers in pharmaceutical factories and institutions engaged in drug study and development.

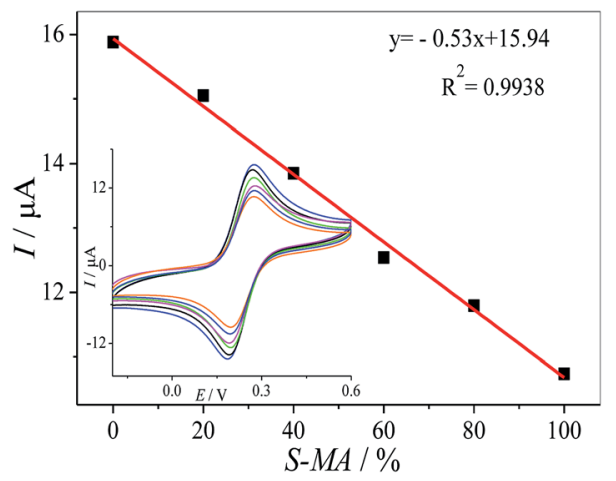

Fig. 12 The linear relationship between $I_{p}$ and the ratio of the concentrations of the MA enantiomers. Inset: $\mathrm{CV}$ of the different ratios of the concentrations of $(S)-M A$ and $(R)-M A(0: 100,20: 80,40: 60$, $60: 40,80: 20$ and $100: 0)$ on the HPCS modified GCE.

\section{Reproducibility and stability}

A pair of modified electrodes was fabricated for discriminating MA enantiomers in repeated tests; the peak current differences were very close to each other and the relative standard deviation (RSD) was 5.19\%. The stability of the prepared chiral sensors was also investigated via $\mathrm{CV}$ measurements over 50 successive scanning cycles in $\left[\mathrm{Fe}(\mathrm{CN})_{6}\right]^{4-/ 3-}$ at $0.5 \mathrm{~V} \mathrm{~s}^{-1}$; the peak current decreased by $0.43 \%$, and it was confirmed that the stability of the MWCNTs-HPCS chiral sensors was fairly satisfactory.

\section{Conclusion}

In summary, MWCNTs-HPCS was prepared via covalent bonds for an effective chiral interface, and the water solubility of HPCS was improved as a result of the introduction of the hydroxypropyl groups, which was applied to the enantioselective recognition of MA enantiomers. The electrochemical recognition of MA enantiomers was obtained at the MWCNTs-HPCS based chiral interface with a peak current ratio of 1.48 . The steric hindrance of HPCS plays an important role during the formation of hydrogen bonds between the MA enantiomers and HPCS. Moreover, the molecular structure of the enantiomers was a key factor in the enantioselective recognition process; for example, the distance between the functional group and chiral carbon, steric hindrance and the type of functional group, and thus, functionalized CS may open up a new way for the fabrication of chiral interfaces used for electrochemical recognition.

\section{Acknowledgements}

We are grateful for financial support from the National Natural Science Foundation of China (51262027), the Natural Science Foundation of Gansu Province (0803RJZA009), Science and Technology Tackle Key Problem Item of Gansu Province (2GS064-A52-036-08), Gansu Key Laboratory of Polymer Materials (ZD-04-14) and the support from the fund of the State Key Laboratory of Solidification Processing in NWPU (SKLSP201011).

\section{Notes and references}

1 R. I. Stefan van Staden, R. G. Bokretsion, K. I. Ozoemena, J. F. van Staden and H. Y. Aboul-Enein, Electroanalysis, 2006, 18, 1718. 
2 A. Zehnacker and M. A. Suhm, Angew. Chem., Int. Ed., 2008, 47, 6970 .

3 C. Z. Xuan, Q. Xia, J. J. Xu, Q. H. Wang, X. Lin and Y. Z. Fu, Anal. Methods, 2016, 8, 3564.

4 B. D. Liu, X. Zhang, Y. P. Ding, L. Q. Luo and F. F. Zhang, Anal. Methods, 2015, 7, 3022.

5 Q. Zhang, L. J. Guo, Y. H. Huang, Y. H. Wang, Q. Han and Y. Z. Fu, Anal. Methods, 2013, 5, 4397.

6 E. Zor, I. H. Patir, H. Bingol and M. Ersoz, Biosens. Bioelectron., 2013, 42, 321.

7 Q. Zhang, Y. H. Huang, L. J. Guo, C. Chen, D. M. Guo, Y. Chen and Y. Z. Fu, New J. Chem., 2014, 38, 4600.

8 L. P. Bao, J. Y. Dai, L. Yang, J. F. Ma, Y. X. Tao, L. H. Deng and Y. Kong, J. Electrochem. Soc., 2015, 162, H486.

9 W. L. Feng, C. Liu, S. Y. Lu, C. Y. Zhang, X. H. Zhu, Y. Liang and J. M. Nan, Microchim. Acta, 2014, 181, 501.

10 D. M. Guo, P. Y. Ran, C. Chen, Y. Chen, C. Z. Xuan and Y. Z. Fu, J. Electrochem. Soc., 2015, 162, B354.

11 J. Ou, Y. X. Tao, J. J. Xue, Y. Kong, J. Y. Dai and L. H. Deng, Electrochem. Commun., 2015, 57, 5.

12 N. M. Alves and J. F. Mano, Int. J. Biol. Macromol., 2008, 43, 401.

13 X. K. Lu, S. J. Tang, B. L. Huang, X. Y. Shen and F. Hong, Fibers Polym., 2013, 14, 935.

14 C. K. S. Pillai, W. Paul and C. P. Sharma, Prog. Polym. Sci., 2009, 34, 641.

15 L. Y. Yu, Q. Liu, X. W. Wu, X. Y. Jiang, J. G. Yu and X. Q. Chen, RSC Adv., 2015, 5, 98020.

16 X. G. Gu, Y. X. Tao, Y. Pan, L. H. Deng, L. P. Bao and Y. Kong, Anal. Chem., 2015, 87, 9481.

17 Y. G. Wang, L. Shi, L. Gao, Q. Wei, L. M. Cui, L. H. Hu, L. G. Yan and B. Du, J. Colloid Interface Sci., 2015, 451, 7.

18 L. P. Bao, Y. X. Tao, X. G. Gu, B. Z. Yang, L. H. Deng and Y. Kong, Electrochem. Commun., 2016, 64, 21.

19 K. W. Tang, J. M. Yi, K. L. Huang and G. L. Zhang, Chirality, 2009, 21, 390.
20 Y. Z. Fu, L. L. Wang, Q. Chen and J. Zhou, Sens. Actuators, B, 2011, 155, 140.

21 E. Zora, A. O. Safc and H. Bingolc, Anal. Biochem., 2014, 449, 83.

22 M. Z. Sai, S. L. Zhong, Y. Tang, W. T. Ma, Y. G. Sun and D. R. Ding, J. Appl. Polym. Sci., 2014, 131, 40535.

23 Y. H. Huang, D. M. Guo, Q. Zhang, L. J. Guo, Y. Chen and Y. Z. Fu, RSC Adv., 2014, 4, 33457.

24 D. M. Guo, P. Y. Ran, C. Chen, Y. Chen, C. Z. Xuan and Y. Z. Fu, J. Electrochem. Soc., 2015, 162, B354.

25 C. S. Wu and H. T. Liao, Polymer, 2007, 48, 4449.

26 Y. Z. Zhang, H. Y. Ma, K. Y. Zhang, S. J. Zhang and J. Wang, Electrochim. Acta, 2009, 54, 2385.

27 C. L. Chen, A. Ogino, X. K. Wang and M. Nagatsu, Appl. Phys. Lett., 2010, 96, 131504.

28 S. Y. Yang, C. C. M. Ma, C. C. Teng, Y. W. Huang, S. H. Liao, Y. L. Huang, H. W. Tien, T. M. Lee and K. C. Chiou, Carbon, 2010, 48, 592.

29 Q. Bi, S. Q. Dong, Y. M. Sun, X. Q. Lu and L. Zhao, Anal. Biochem., 2016, 508, 50.

30 H. Gou, J. X. He, Z. L. Mo, X. J. Wei, R. R. Hu, Y. W. Wang and R. B. Guo, J. Electrochem. Soc., 2016, 163, B272.

31 D. M. Guo, Y. H. Huang, C. Chen, Y. Chen and Y. Z. Fu, New J. Chem., 2014, 38, 5880.

32 J. J. Xu, Q. H. Ying, Q. Xia, N. Wang, X. Lin and Y. Z. Fu, New J. Chem., 2016, 40, 6955.

33 L. P. Bao, X. H. Chen, B. Z. Yang, Y. X. Tao and Y. Kong, ACS Appl. Mater. Interfaces, 2016, 8, 21710.

34 Y. X. Tao, J. Y. Dai, Y. Kong and Y. Sha, Anal. Chem., 2014, 86, 2633.

35 F. H. Stillinger and A. Rahman, J. Chem. Phys., 1972, 57, 1281.

36 T. I. Mizan, P. E. Savage and R. M. Ziff, J. Phys. Chem., 1996, 100, 403.

37 G. R. Desiraju, Acc. Chem. Res., 1996, 29, 441.

38 L. L. Wang, W. C. Gong, F. Wang, Z. Y. Yu and Z. L. Chen, Anal. Methods, 2016, 8, 3481. 